INTERNATIONAL JOURNAL OF MULTIDisciplinARY RESEARCH AND ANALYSis

ISSN(print): 2643-9840, ISSN(online): 2643-9875

Volume 04 Issue 06 June 2021

DOI: 10.47191/ijmra/v4-i6-14, Impact Factor: 6.072

Page No.- 774-778

\title{
The Study of Dielectric Behavior and Phase Transition of Mixed \\ Compound of Lead Tantalate Sintered with Silver Oxide with
}

\section{Temperature Variation}

\author{
Shri Prakash Dubey ${ }^{1}$, Subhash Kumar Sharma ${ }^{2}$ \\ ${ }^{1}$ Department of Physics, M. G. P. G. College, Gorakhpur, UP, India. \\ ${ }^{2}$ Department of Electronics, M. G. P. G. College, Gorakhpur, UP, India
}

\begin{abstract}
The dielectric constant of mixed compound of Lead Tantalate $\left(\mathrm{PbTa}_{2} \mathrm{O}_{6}\right)$ sintered with Silver oxide has been measured between the temperatures $40^{\circ} \mathrm{C}$ to $300^{\circ} \mathrm{C}$ using the capacitance bridge model ZENITH M $92 \mathrm{~A}$ at the frequency of $1000 \mathrm{~Hz}$. During the measurement, it is found that the properly annealed sample have three peaks at the temperature $90^{\circ} \mathrm{C}(\varepsilon=1000), 220^{\circ} \mathrm{C}(\varepsilon$ $=800)$ and $230^{\circ} \mathrm{C}(\varepsilon=623)$ respectively and a high peak at the temperature of $130^{\circ} \mathrm{C}(\varepsilon=1600)$ in the heating cycle curve with some intermediate fluctuations. When the variation of dielectric constant was studied in cooling cycle the peak was observed at 240 ㅇ $\mathrm{C}$, above and below this temperature, dielectric constant decreases with some intermediate fluctuations showing shows a first order phase transition. The variation in heating cycle is explained on the basis of activated polarization process. When the behavior of compound is studied in cooling cycle, then pronounced hysteresis is found with certain intermediate fluctuations. The cooling cycle curve does not follow heating curve because of the temperature relaxation of the compound. The occurrence of peak at the Curie temperature is explained by the latent heat type properties of the mixed compound. The temperature variation of the mixed compound suggests for its valuable applications as active and smart materials and its applications in the ceramics.
\end{abstract}

KEYWORDS: Dielectric Constant, Phase Transition, Curie temperature, Polarization and Ceramics.

\section{INTRODUCTION: Ferro Electric Curie point And Phase Transition}

An important characteristic of ferroelectrics is the temperature of phase transition called the Curie point $\Theta_{c}$. When the temperature decreases through the Curie point, a ferroelectric crystal undergoes a structural phase transition from a paraelectric phase to a ferroelectric phase. When the temperature is above $\Theta_{c}$ the crystal does not exhibit Ferro electricity, on the other hand, when the temperature is below $\Theta_{c}$, the crystal exhibits Ferro electricity. It is generally believed that the ferroelectric structure of a crystal is created by a small distortion of the paraelectric structure such that the lattice symmetry in the ferroelectric phase is always lower than that in the par electric phase. If there are two, or more ferroelectric phases in a crystal, the Curie point only specifies the temperature at which a paraelectric-ferroelectric phase transition occurs. A temperature, at which the crystal transforms from one ferroelectric phase to another ferroelectric phase, is always called a transition temperature [1].

For example, the Curie point of $\mathrm{BaTiO}_{3}$ is $120^{\circ} \mathrm{C}$, at which the paraelectric-ferroelectric phase transition occurs. However, there are three ferroelectric phases in $\mathrm{BaTiO}_{3}$ below $120^{\circ} \mathrm{C}$ [2]. The phase transition temperature from one ferroelectric phase to another, i.e., $0^{\circ} \mathrm{C}$ and $-80^{\circ} \mathrm{C}$, are not called Curie points. Both Rochelle salt (RS) and deuterated RS possess two Curie points[3], and their ferroelectric phases exist between the higher Curie point $\left(24^{\circ} \mathrm{C}\right)$ and the lower Curie point $\left(-18^{\circ} \mathrm{C}\right)$. Some ferroelectric crystals, such as GASH (guanidine aluminum sulfate hexahydrate) [3], $\mathrm{LiH}_{3}\left(\mathrm{SeO}_{3}\right)_{2}$ (lithium trihydrogen selenite) [4] and BaCoF 4 family [5], etc. do not possess Curie points owing to the fact that they remain ferroelectric up to their decomposition temperatures.

When the temperature is in the vicinity of the Curie point, thermodynamic properties (such as dielectric, elastic, optical, and thermal properties) of a ferroelectric crystal show anomalies and the structure of the crystal changes. For example, the dielectric constant in most ferroelectric crystals has a very large (abnormal) value (up to $10^{4}-10^{5}$ ) near their Curie points. This phenomenon is usually called the 'dielectric anomaly'. The dielectric constants are reported against the temperature for $\mathrm{BaTiO}_{3}$ [6] and Rochelle salt [7]. In most ferroelectrics, the temperature dependence of the dielectric constant above the Curie point (in 


\section{The Study of Dielectric Behavior and Phase Transition of Mixed Compound of Lead Tantalate Sintered with Silver Oxide with Temperature Variation}

the paraelectric phase regime) can be described fairly accurately by a simple law called the Curie-Weiss law

$$
\varepsilon=\varepsilon_{0}+C / \Theta-\Theta_{0} \quad \Theta>\Theta_{0}
$$

Where $C$ is the Curie-Weiss constant and $\Theta_{0}$ the Curie-Weiss temperature. $\Theta_{0}$ is different from the Curie point $\Theta_{c}$. In the case of a first-order phase transition, $\Theta_{0}<\Theta_{c}$ while for the second-order phase transition $\Theta_{0}=\Theta_{c}$. If we examine the temperature dependence of the dielectric constant, or in other words, the value of the Curie constant $C$ appearing in the Curie-Weiss law, we see that the various ferroelectrics can be divided into two main groups. Compounds in the first group have Curie constants of the order of $10^{3}$. It can be shown that this order of magnitude is to be expected for a substance containing a number of similar dipoles, each of which has two positions of equilibrium corresponding to opposite orientations of the dipole. The dielectric constant $\varepsilon$ of such a model assumes the following form

$$
\varepsilon=\frac{4 \pi}{\gamma} \frac{\mathrm{T}_{0}}{\mathrm{~T}-\mathrm{T}_{0}}
$$

Where $\gamma$ is the Lorentz factor appearing in the Lorentz internal-field formula $F=E+\gamma P$, and $T_{0}$ is the Curie-Weiss temperature. The factor $\gamma$ will depend on the particular atomic structure but will be of the order of $4 \pi / 3$. Thus, we can write for the dielectric constant

$$
\varepsilon \cong \frac{3 \mathrm{~T}_{0}}{\mathrm{~T}-\mathrm{T}_{0}}
$$

Implying that the Curie constant $\mathrm{C}$ is of the order of $3 \mathrm{~T}_{0}$, i.e. for $\mathrm{T}_{0} \sim 300^{\circ} \mathrm{K}$, of the order of $10^{3}$. The second group of ferroelectrics exhibit Curie constants of the order of $10^{5}$. This can be explained by assuming that the substances in this group contain, say, $\mathrm{N}$ oscillating ions per unit volume and that each ion produces an electric moment when it is displaced a distance $\mathrm{x}$ from its normal equilibrium position. Expressing the energy of an ion relative to its equilibrium position in the form $a x^{2}+b x^{4}$, Devonshire has shown that the dielectric constant assumes the form:

$$
\varepsilon=\frac{4 \pi \mathrm{Ne}^{2}}{6 \mathrm{k}} \frac{\mathrm{a}}{\mathrm{b}} \frac{1}{\mathrm{~T}-\mathrm{T}_{0}}
$$

Where $\mathrm{k}$ is Boltzmann's constant. Thus, the temperature dependence of the dielectric constant depends on the anharmonic term $b x^{4}$ in the energy of an ion. This equation shows that if the coefficient $b$ is small, the temperature dependence of $\varepsilon$ is small and hence the Curie constant will be large.

It is evident that the four classifications described above do not coincide with each other. Each one is useful only when discussing a particular aspect of the ferroelectric phenomenon, but a consistent classification of all ferroelectrics appears hardly possible at the present stage [8].

\section{EXPERIMENTAL}

The compound has been procured from E.Merk (India), Mumbai. The chemical was grinded into the fine powder in an agate mortar, avoiding direct sunlight and preferably the most of the sample preparation was done at night. The pellets were prepared with compression machine (Flextural Testing Machine CAT No.AIM-313, S.No.91070 AIMIL Associated, India), having pressure range 0-10 tonne $\mathrm{wt} / \mathrm{cm}^{2}$. A suitable die was used having rectangular Cross-Sectional area of the piston $=2.33 \mathrm{~cm}^{2}$.

The polishing of the pellets has been done to obtain smooth parallel surface to be used for electrode formation polishing of the crystal introduces electrical charges inside the material. These charges and strains are to be removed, which we did by the process of annealing of the sample. In this process the pellets were kept in a suitable furnace at nearly $2 / 3$ of their melting points for sufficient times (generally 8-10 hours). The most of the irreproducibility was removed by annealing and therefore this process was necessarily done. The electrodes were formed using colloidal silver paints.

The sample holder loaded with pellet is kept into the furnace such that it lies very near to the middle part of the furnace. A good quality thermometer, precisely calibrated is used to record the temperature. This thermometer is adjusted with the help of stand in such a way that it touches the metallic part of sample holder to record the exact temperature of sample.

The usual substitution method i.e. with and without the specimen in suitable sample holder is used [9]. The sample holder was directly fastened to the capacity measuring unit (Zenith M 92A). 
The Study of Dielectric Behavior and Phase Transition of Mixed Compound of Lead Tantalate Sintered with Silver Oxide with Temperature Variation

\section{RESULTS AND DISCUSSION}

We have selected the solid solution of the compound Lead Tantalate $\left(\mathrm{PbTa}_{2} \mathrm{O}_{6}\right)$ and Silver Oxide $(\mathrm{AgO})$ in which percentage of $\mathrm{AgO}$ is 33. During the measurement, it is found that the properly annealed sample have three peaks at the temperature $90^{\circ} \mathrm{C}(\varepsilon=1000)$, $220^{\circ} \mathrm{C}(\varepsilon=800)$ and $230^{\circ} \mathrm{C}(\varepsilon=623)$ respectively and a high peak at the temperature of $130^{\circ} \mathrm{C}(\varepsilon=1600)$ in the heating cycle curve. The heating cycle curve is shown in the Fig.1.

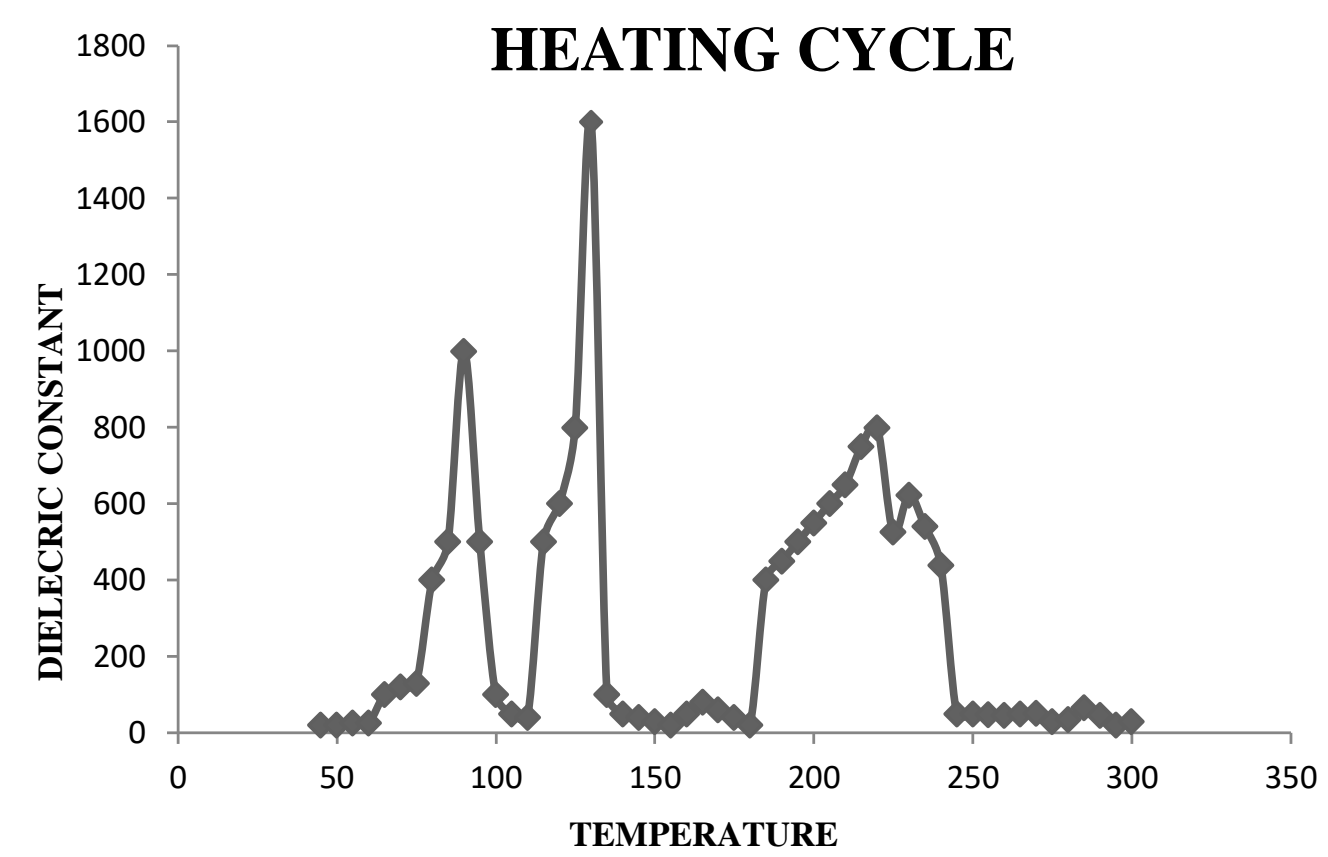

Fig 1. Temperature Variation Of Dielectric Constant Of Lead Tantalate Sintered With Silver Oxide

Lead Tantalate $\left(\mathrm{PbTa}_{2} \mathrm{O}_{6}\right)$ was reported to be ferroelectric by means of ceramic specimens. The Curie point lies at $230^{\circ} \mathrm{C}[10]$. The Curie constant has the value $1.5 \times 10^{5}$ degrees and the spontaneous polarization of mixed compound is equal to $10 \times 10^{-6} \mathrm{C} / \mathrm{cm}^{2}$ at room temperature. The experimentally, the ferroelectric phase of Lead Tantalate $\left(\mathrm{PbTa}_{2} \mathrm{O}_{6}\right)$ with silver oxide was obtained only by properly annealed and heated up to $300^{\circ} \mathrm{C}$. When prepared below this temperature the compound is rhombohedra [11]. The ferroelectric phase is orthorhombic, and the lattice parameters as measured are

$a=17.68 \AA, b=17.72 \AA, c=2 \times 3.877 \AA$

Indicating a small orthorhombic distortion $\mathrm{b} / \mathrm{a}=1.002$. Previous studies of polycrystalline $\mathrm{PbTa}_{2} \mathrm{O}_{6}$ had led to tetragonal symmetry [12]. Further investigation has done that this mixed compound approaches tetragonality with increasing temperature but does not attain it. Above the Curie temperature, the structure is still orthorhombic although different, of course, from that of the ferroelectric phase. So the polar axis can have only two antiparallel orientations, in accordance with the biaxial nature of the nonferroelectric phase. Thus, the [100] and [010] axes are not interchangeable under the action of external electric fields and polarization reversal occurs only through $180^{\circ}$ motion [13].

The curve for the cooing cycle is shown in the Fig.2. The cooling does not follow heating curve because of the temperature relaxation of the compound [14]. 


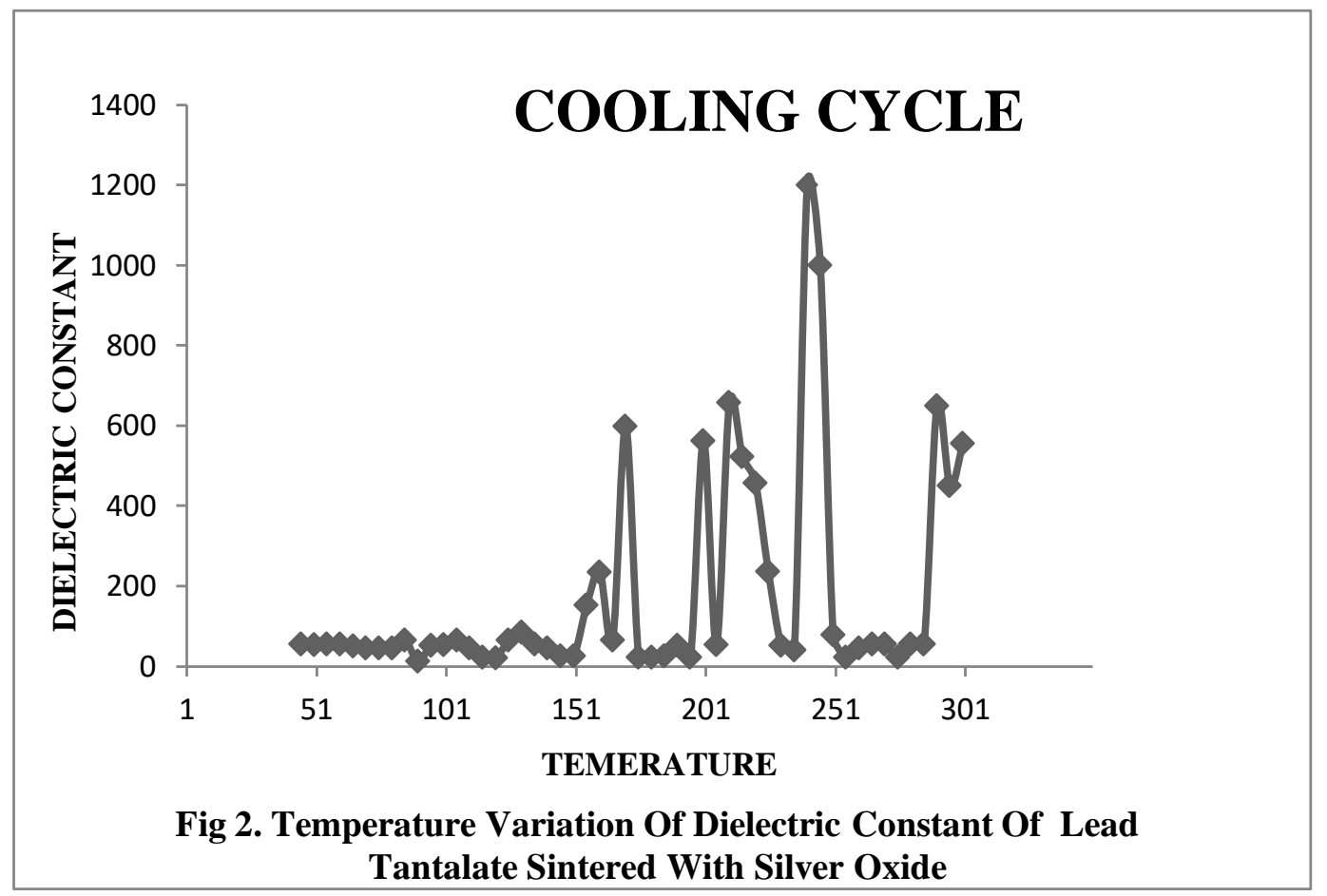

As temperature is increased from room temperature the dipoles got sufficient kinetic energy to face hindrance, in rotation and alignment. The dielectric constant and the alignment of dipoles could not achieve the previous high value of dielectric constant at the moderate temperature of $130{ }^{\circ} \mathrm{C}$ in cooling cycle. In the same cycle, the dielectric constant has its highest value again, in the same range of temperature at $240^{\circ} \mathrm{C}$, which is moderately different than that of heating cycle. The peak value of the dielectric constant at the Curie temperature as observed in our sample (Fig.2) and the shape of the anomaly are strongly dependent on the rate of temperature change during the measurement. The shape of the anomaly depicted in cooling cycle is obtained with very slow cooling rates and due to the structural phase change in the crystal. The fluctuation in the variation of dielectric constant with temperature is due to the rotation of silver ions in the crystal lattice of the compound. Similar explanation has been given for the variation of dielectric constant near Curie temperature in the case of Lead Nitrate [15] and Barium Nitrate [16].

\section{CONCLUSION}

In this work we have concluded that the change in temperature changes the structure causing the phase change in the compound and dipoles are created due to the active silver ion get sufficient kinetic energy to face hindrance. The rotation and alignment of the dipoles shows the Ferro electricity in the compound.

\section{ACKNOWLEDGEMENT}

The authors are thankful to Head, Department of Physics, HNB GARHWAL University, Srinagar, Garhwal, Head, Department of Physics, D.D.U. Gorakhpur University, Gorakhpur and The Principal, MGPG College Gorakhpur for providing experimental facilities and their encouragement.

\section{REFERENCES}

1) Jeludev I.S., 'Ferro electricity and Symmetry', in: "Solid State Physics", Academic Press., N.Y., 26, 1971.

2) Holden A.N., Matthias B.T., Merz W.J. and Remeika J.P, Phys.Rev, 98, p.546, 1955.

3) Pepinsky R and Vedam K., Phys.Rev, 114, p.1217, 1959.

4) Eibschutz $M$ and Guggenheim H.J., Solid.State.Commun, 6, p.737, 1968.

5) Merz W.J., J.Appl.Phys, 27, p.938, 1956.

6) Hablutzel J., Helv.Phys.Acta, 12, p.489, 1939.

7) Jona F and Shirane G., 'Classification of Ferroelectrics', in: “Ferroelectrics Crystals”, (Pergamon Press, Macmillan.Co., N.Y.,), p.11-14, 1962.

8) Gupta R.N and Misra M., Indian J. Pure Appl. Phys, 19, p.1151, 1981.

9) Isupov V.A., Fiz. Tverdogo. Tela1, 242, 1959. 
The Study of Dielectric Behavior and Phase Transition of Mixed Compound of Lead Tantalate Sintered with Silver Oxide with Temperature Variation

10) Subbarao E.C., Shirane G and Jona F., Acta Cryst, 13, p.226, 1960.

11) Francombe M. H and Lewis B., Acta. Cryst. 11, p. 696, 1958.

12) Ismailzade I G., Kristallografiya, 4, p.658, 1959.

13) J.A. Gonjalo., Ferro electricity (The Fundamental Collection); Theory of Ferroelectrics, Wiley-VCH Verlag Gmbh \& Co.Kgaa, P. 149, 2005.

14) Weider H. H., J.Appl.Phys, 40(30), p.1010, 1998.

15) Yadava S., Pandey B. K., Dubey S. P and Gupta R. N., Asian J. Chem., 20(1), p.731-735, 2008.

16) Pandey B. K., Dubey S. P and Gupta G. K., International Journal Mat. Sci. Res. India, 7(1), p.307-310, 2010. 